\title{
Contralateral bulla neogenesis associated with postoperative recurrences of primary spontaneous pneumothorax in young patients
}

\author{
Kenji Tsuboshima*, Yasumi Matoba, Teppei Wakahara \\ Department of Thoracic Surgery and Pneumothorax Center, Takasago Municipal Hospital, Takasago, Japan \\ Contributions: (I) Conception and design: K Tsuboshima; (II) Administrative support: Y Matoba; (III) Provision of study materials or patients: K \\ Tsuboshima; (IV) Collection and assembly of data: All authors; (V) Data analysis and interpretation: Kenji Tsuboshima; (VI) Manuscript writing: All \\ authors; (VII) Final approval of manuscript: All authors. \\ Correspondence to: Kenji Tsuboshima, MD. Pneumothorax Research Center and Division of Thoracic Surgery, Nissan Tamagawa Hospital, 4-8-1 Seta \\ Setagaya-ku, Tokyo 158-0095, Japan. Email: drniwatori@smn.enjoy.ne.jp.
}

\begin{abstract}
Background: Video-assisted thoracoscopic surgery (VATS) is considered an acceptable treatment for recurrent primary spontaneous pneumothorax (PSP). However, recent reports have described a high postoperative recurrence rate in young patients with PSP. We hypothesized that ineffective VATS may have been performed for these patients (aged $<25$ years). We evaluated the factors preventing postoperative recurrence in young PSP patients in order to identify patients with effective surgery.

Methods: Between April 2009 and December 2018, we retrospectively evaluated 92 patients aged $<25$ years who underwent first VATS bullectomy for PSP. Factors such as gender, smoking habit, history of contralateral PSP, family history, body mass index (BMI), and contralateral bulla neogenesis on highresolution computed tomography were evaluated.

Results: The mean BMI was 18.8 \pm 1.8 . Contralateral bulla neogenesis was present in 31 patients $(33.7 \%)$. The median period of observation for postoperative recurrence was 401.5 days. Thirty cases $(32.6 \%)$ developed postoperative recurrence. Univariate and multivariable analyses showed that a $\mathrm{BMI} \geq 18.0$ and no contralateral bulla neogenesis were significant factors preventing postoperative recurrence $(\mathrm{P}=0.018$, hazard ratio: 0.41 and $\mathrm{P}=0.0046$, hazard ratio: 0.25 , respectively).

Conclusions: Although the patients aged $<25$ years have a high rate of postoperative recurrence, the patients with $\mathrm{BMI} \geq 18.0$ and no contralateral bulla neogenesis have a significantly lower rate of postoperative recurrence rate.
\end{abstract}

Keywords: Primary spontaneous pneumothorax (PSP); bulla neogenesis; young; postoperative recurrence

Submitted Jul 29, 2019. Accepted for publication Nov 12, 2019.

doi: $10.21037 /$ jtd.2019.12.05

View this article at: http://dx.doi.org/10.21037/jtd.2019.12.05

\section{Introduction}

Primary spontaneous pneumothorax (PSP) is a common disease with a relatively high recurrence rate, and cases of severe PSP are rare. In particular, young healthy men with no apparent underlying pulmonary disease suffer from PSP. Video-assisted thoracoscopic surgery (VATS) bullectomy is an acceptable treatment for cases of recurrent PSP (1-3). However, it has recently been suggested that younger patients have a higher postoperative recurrence (21.9-27.9\%) of pneumothorax than older patients, even if the same procedures for prevention of the postoperative

\footnotetext{
* Pneumothorax Research Center and Division of Thoracic Surgery, Nissan Tamagawa Hospital, Tokyo, Japan at present.
} 


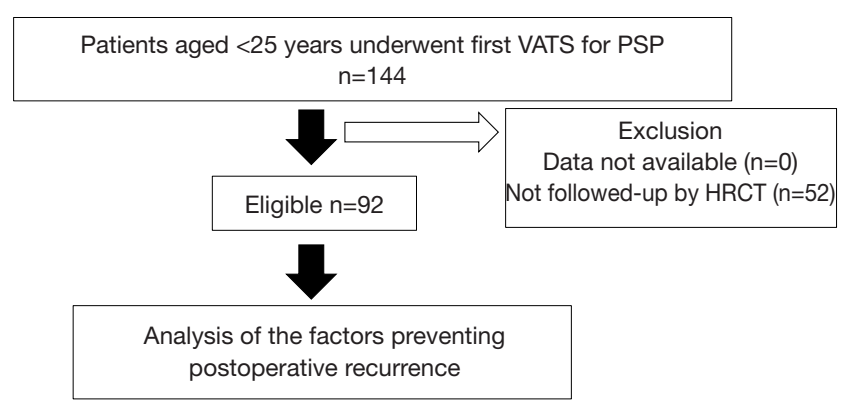

Figure 1 Characteristics of patients enrolled in the study. HRCT, high-resolution computed tomography; PSP, primary spontaneous pneumothorax; VATS, video-assisted thoracoscopic surgery.

recurrence are performed (4-7). Bulla neogenesis is thought to be the main reason for postoperative recurrence $(8-10)$. In addition, it was reported that young patients aged $<20$ years frequently developed bulla neogenesis regardless of surgical treatment (11). These results may reflect a relationship between frequent postoperative recurrence and bulla neogenesis for young patients with PSP.

Although there are guidelines for the management of PSP $(1,2)$ based on various conditions, such as radiological findings or air leakage, there are no guidelines for the age-related management of PSP. The aim of this study was to identify the factors that can prevent postoperative recurrence of PSP in young individuals. We retrospectively evaluated the factors preventing postoperative recurrences including bulla neogenesis, in patients aged $<25$ years who underwent VATS bullectomy.

\section{Methods}

A total of 144 consecutive patients with PSP aged $<25$ years who underwent first VATS procedure in our hospital were enrolled between April 2009 and December 2018. The exclusion criterion was the unavailability of high-resolution computed tomography (HRCT) data for the preoperative or postoperative investigation. After exclusion, 92 patients were enrolled (Figure 1). Factors preventing postoperative recurrence were retrospectively evaluated using the Kaplan-Meier method and Cox regression analysis. Factors preventing postoperative recurrence were as follows: gender, body mass index (BMI), smoking habit, history of contralateral PSP, family history, and bulla neogenesis.

Patients underwent VATS according to PSP management guidelines because of the following indications: ipsilateral recurrence of PSP, a prolonged air leak after chest tube drainage, bilateral pneumothorax, and patient wishes
$(1,2)$. Persistent air leak was defined as air leak for a period of over 7 days. As previously described (12), the patient was placed in a lateral position under general anesthesia with the ventilation of one lung. The target lesions were determined based on preoperative computed tomographic and intraoperative thoracoscopic findings, including an air leak test under $20 \mathrm{cmH}_{2} \mathrm{O}$ of pressure using sterile, distilled water. The target lesions, including the bulla and thickened pleura, were resected with a sufficient margin, using $45-\mathrm{mm}$ cartridge autosutures, and no residual lesions were identified intraoperatively. The staple line was reinforced with regenerated oxidized cellulose and/or fibrin glue, depending on the situation. The choice of procedure (single-port or multi-port VATS) was made based on surgical difficulties which were affected by the number or site of lesions and extent of intrathoracic adhesion.

Preoperative HRCT was performed to detect the location of the lesions, and postoperative HRCT was performed to determine the presence of bulla neogenesis or PSP recurrence after 12 postoperative months, even in asymptomatic patients. Patients who had symptoms visited our institution without any hesitation, and HRCT was performed, as necessary. Bulla neogenesis was defined as the development or increase of the contralateral bulla against the ipsilateral VATS side based on changes in the preoperative and postoperative HRCT images (Figure 2). The follow-up period for evaluation of bulla neogenesis was defined as the time between the preoperative and postoperative HRCT evaluation. In cases of persistent increase of the bulla on postoperative HRCT at several time points, the period of the follow-up that revealed the first appearance or increase of the bulla was defined as the follow-up period.

Postoperative recurrence was defined as ipsilateral lung collapse on chest radiography or HRCT after postoperative day 29, regardless of treatment necessity. The follow-up period of surgery was defined as the period between the date of operation and the date of censor or recurrence. Censor was defined as the date of the final visit for patients without recurrences. The date of recurrence was defined as the date of the first postoperative recurrence. The postoperative recurrence rate for each variable was calculated as the number of recurrences per objects. According to the Japan Clinical Oncology Group's postoperative complication criteria based on the Clavien-Dindo classification, morbidity or mortality for our procedures was evaluated (13). The definition of severe morbidity was adverse events that corresponded to grade IIIB or more in our previous reports 

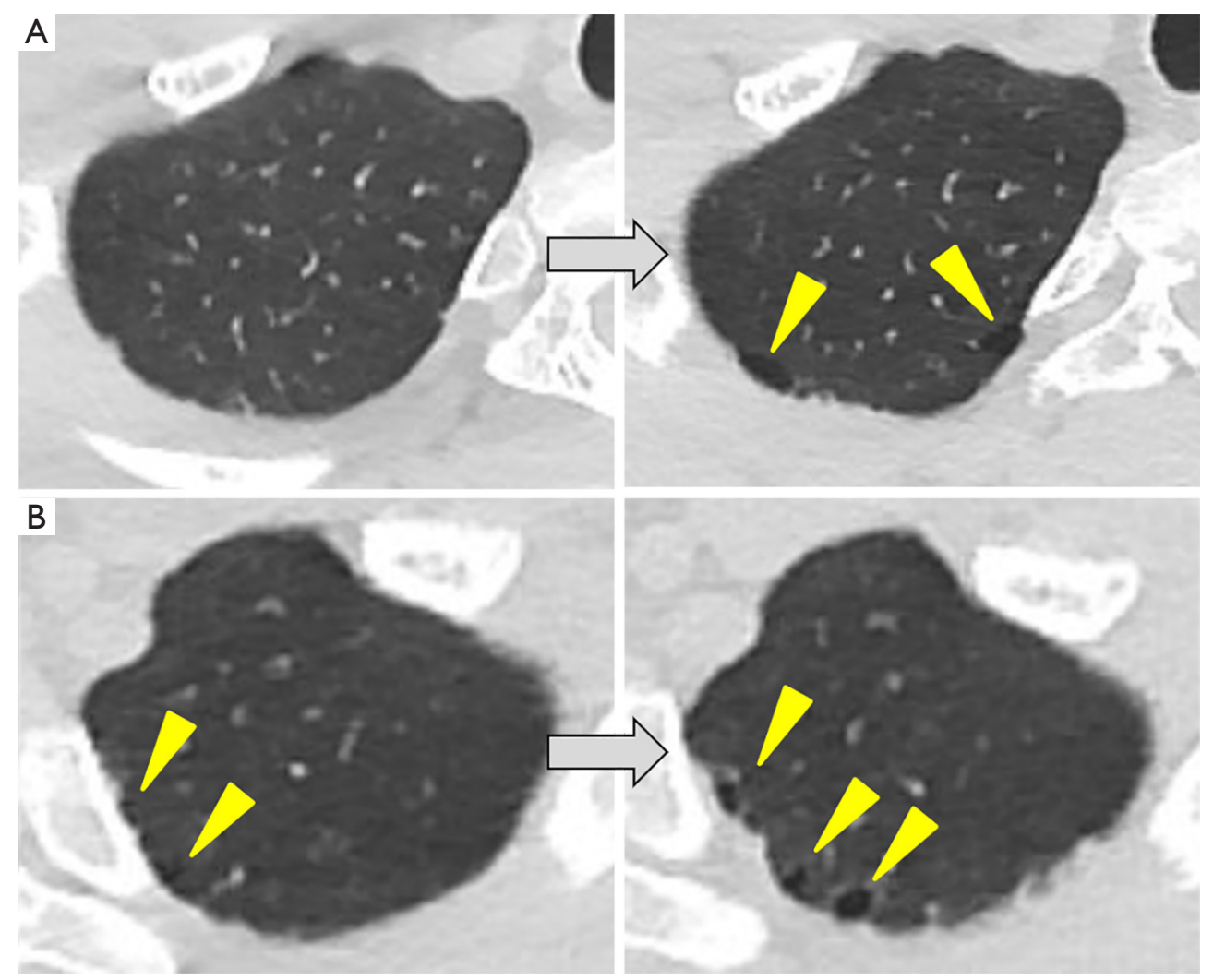

Figure 2 Definition of bulla neogenesis based on high-resolution computed tomography (yellow allows shows bullae). (A) Appearance of new bulla; (B) increase in bulla.

(7,10-12). The postoperative complication was defined as ipsilateral lung collapse within 28 postoperative days.

For statistical analyses, continuous variables were presented as mean \pm standard deviation or median with range. Univariate analysis of the postoperative recurrence rate of PSP was estimated based on the Kaplan-Meier method and was compared using the log-rank test. For timedependent factors such as contralateral bulla neogenesis, the Cox regression analysis was used for the univariate analysis. Multivariable analysis of the postoperative recurrence rate of PSP was evaluated according to Cox regression analysis using all factors by stepwise method. The validity of the model was estimated by the likelihood ratio test, Wald test, and score test. A P value of $<0.05$ was considered statistically significant. The hazard ratio and $95 \%$ confidence intervals (CI) were applied to analyze the preventing factors for postoperative recurrence. The data were analyzed using the statistical software EZR package (http://www.jichi.ac.jp/ saitama-sct/SaitamaHP.files/statmed.html).

The Institutional Review Board of Takasago Municipal Hospital approved this study (No. 2018-11), and we obtained informed consent from each patient by optout.

\section{Results}

The mean age was $18.4 \pm 2.5$ years, with male predominance $(\mathrm{n}=84,91.3 \%)$ (Table 1$)$. The mean BMI was $18.8 \pm 1.8$ (Table 1). A smoking habit was present in 12 patients $(13.0 \%)$ (Table 1). The median number of pre- and postoperative contralateral bullae was 1 [0-13] and 2 [0-11], respectively. Thirty-one patients had bulla neogenesis (33.7\%). The median follow-up period for the evaluation of bulla neogenesis was 409 days (Table 1). The median followup period for the evaluation of postoperative recurrence was 401.5 days (Table 1). The main indications for surgery were ipsilateral recurrence $(n=45,48.9 \%)$, prolonged air leakage $(n=20,21.7 \%)$, patient request $(n=16,17.4 \%)$, bilateral pneumothorax $(\mathrm{n}=6,6.5 \%)$, and others $(\mathrm{n}=5,5.4 \%)$.

According to the univariate analysis, two factors including BMI $\geq 18.0$ and no bulla neogenesis significantly reduced postoperative recurrence (Table 2). Based on Cox regression analysis using all factors by stepwise method, BMI $\geq 18.0$ 
Table 1 Clinical characteristics of PSP patients who underwent VATS

\begin{tabular}{lc}
\hline Variables & Clinical characteristics $(\mathrm{n}=92)$ \\
\hline Age (years) & $18.4 \pm 2.5$ \\
Male, $\mathrm{n}(\%)$ & $84(91.3)$ \\
BMI & $18.8 \pm 1.8$ \\
Smoking history (present), $\mathrm{n}(\%)$ & $12(13.0)$ \\
History of contralateral PSP (present), $\mathrm{n}(\%)$ & $11(12.0)$ \\
Family history (present), $\mathrm{n}$ (\%) & $15(16.3)$ \\
Number of preoperative bullae, median [range] & $1[0-13]$ \\
Number of postoperative bullae, median [range] & $2[0-11]$ \\
Bulla neogenesis, $\mathrm{n}$ (\%) & $31(33.7)$ \\
Postoperative recurrence, $\mathrm{n}(\%)$ & $30(32.6)$ \\
Follow-up period of bulla neogenesis (days), median [range] & $409[2-3,166]$ \\
Follow-up period of postoperative recurrence (days), median [range] & $401.5[16-3,339]$ \\
\hline
\end{tabular}

BMI, body mass index; PSP, primary spontaneous pneumothorax; VATS, video-assisted thoracoscopic surgery.

Table 2 Univariate analysis to assess factors preventing postoperative recurrence in PSP patients

\begin{tabular}{lcc}
\hline Variables & Postoperative recurrence rate (\%) & P values \\
\hline Gender (male vs. female) & 34.5 vs. 12.5 & 0.49 \\
BMI (<18 vs. $\geq 18)$ & 45.5 vs. 25.4 & 0.036 \\
Smoking habit (absent vs. present) & 35.0 vs. 16.7 & 0.096 \\
History of contralateral PSP (absent vs. present) & 33.3 vs. 27.3 & 0.93 \\
Family history (absent vs. present) & 33.8 vs. 26.7 & 0.56 \\
Bulla neogenesis (absent vs. present) & 21.3 vs. 54.8 & 0.013 \\
\hline
\end{tabular}

BMI, body mass index; PSP, primary spontaneous pneumothorax.

and no bulla neogenesis were significant factors preventing postoperative recurrence $[\mathrm{P}=0.018$; hazard ratio: $0.41(95 \%$ CI: 0.195-0.861) and $\mathrm{P}=0.0046$; hazard ratio: 0.25 (95\% CI: 0.096-0.651), respectively] (likelihood ratio test: $\mathrm{P}=0.0043$; Wald test: $\mathrm{P}=0.0029$; score test: $\mathrm{P}=0.018$ ) (Table 3). For two factors, an assumption of the proportional hazards was confirmed. No severe morbidity or mortality was found.

\section{Discussion}

Until recently, surgical treatment for PSP has been thought to have a lower recurrence rate $(1.3-16.5 \%)$ $(1-3,4-6)$ than conservative therapies $(29.2-54.2 \%)(14,15)$. Therefore, surgical treatment such as VATS bullectomy has been considered an acceptable treatment for patients with recurrent PSP (1-3). However, it has recently been revealed that younger patients had a higher postoperative recurrence rate than elderly patients, even if underwent the same procedures intraoperatively (4-7). Nakayama et al. described that younger patients had high recurrence rate after VATS bullectomy with an absorbable polyglycolic acid sheet. Younger patients aged $<23$ years had a significantly lower 5 -year postoperative recurrence-free probability than those aged $\geq 23$ years ( $78.1 \%$ vs. $93.8 \%$ ) (4). Huang et al. performed a multivariable analysis and demonstrated that younger age was affected to a significant postoperative recurrence after VATS bullectomy with mechanical or chemical pleurodesis (5). Noh et al. showed that younger patients aged $\leq 16$ years had high recurrence rate after a wedge resection than in elderly patients, significantly. 
Table 3 Cox regression analysis to assess factors preventing postoperative recurrence in PSP patients

\begin{tabular}{llll}
\hline Variable & HR & $95 \% \mathrm{Cl}$ & $\mathrm{P}$ value \\
\hline $\mathrm{BMI} \geq 18$ & 0.41 & $0.195-0.861$ & 0.018 \\
Bulla neogenesis present & 0.25 & $0.096-0.651$ & 0.0046 \\
\hline
\end{tabular}

$\mathrm{Cl}$, confidence interval; HR, hazard ration; PSP, primary spontaneous pneumothorax.

However, there was no difference in the recurrence rates after observation or closed thoracostomy, regardless of age (45.7-51.9\%) (6). In our previous study, we reported that young patients aged $<25$ years who underwent VATS bullectomy with pleural coating using $50 \%$ glucose solution had a significant postoperative recurrence factor in univariate analysis (7). The current study has adopted an age cut-off of 25 years, similar to the aforementioned reports (4-7).

Bulla neogenesis is thought to be the main reason for postoperative recurrence (8-10). Muramatsu et al. reported that the new bulla formation was the most common recurrence factor after VATS bullectomy alone for PSP (8). Cho et al. observed that the most common reason for recurrent PSP after VATS was the formation of a bulla on the previous staple line, followed by a new bulla formation in a location other than the staple line (9). In our previous study, younger patients aged $<25$ exhibited a higher tendency for detection of postoperative bulla neogenesis on the staple line using HRCT, significantly (10). In addition, we reported that bulla neogenesis had a significantly higher prevalence rate in patients aged $<20$ years than in patients aged $\geq 20$ years (44.8\% vs. $8.2 \%)$ using univariate, multivariable, and propensity score analyses for 155 patients with PSP as the natural history (11). These results (8-11) may show a relationship between frequent postoperative recurrence and bulla neogenesis in young patients with PSP. However, it is difficult to precisely evaluate bulla neogenesis of the ipsilateral side using pre- or postoperative HRCT due to various changes such as lung collapse, surgical procedures, and adhesion. Therefore, in this study, the contralateral lung, which had not altered because of lung collapse or operation was investigated. We hypothesized that the ipsilateral and contralateral lung have the same trend for bulla neogenesis. We thought that HRCT findings of contralateral lung bulla neogenesis could predict ipsilateral lung bulla neogenesis and postoperative recurrence for young patients with PSP. Therefore, we considered contralateral bulla neogenesis, which was observed on HRCT for approximately 1 year (median follow- up duration: 409 days), as one of the factors preventing postoperative recurrence. In fact, our study showed that no contralateral bulla neogenesis is a factor preventing postoperative recurrence for patients with PSP.

Our study showed that BMI $\geq 18$ was also a significant factor preventing postoperative recurrence. Tan et al. reported an association between BMI and PSP recurrence (16). They found low BMI to be a significant predictor [hazard ratio: 3.668 (95\% CI: $1.632-7.145$ ), $\mathrm{P}<0.001]$ of PSP recurrence among 273 patients on multivariable analysis. They provided several possible explanations for this association, such as unbalanced physical development, deficiency in $\alpha 1$-antitrypsin, and abnormal bone mineral density. Additionally, Onuki et al. described that a history of contralateral PSP was a significant risk factor for postoperative recurrence in patients aged $\leq 20$ years using univariate analysis (17). They evaluated 66 cases, $36(54.5 \%)$ of which had been followed-up for only 1 year. We did not obtain the same result, which may be explained by the small number of cases and relatively short period of follow-up in the study by Onuki et al. (17).

Our study has some limitations. We performed a retrospective study with a small sample size; therefore, a prospective study with a large sample size may be required for the management of young patients with recurrent PSP. Although we described that surgical treatment may be considered in young patients aged $<25$ years with $\mathrm{BMI} \geq 18$ and no bulla neogenesis who had a recurrence after the first conservative therapy, no direct comparison between the recurrence rates after surgery and after second conservative therapy was performed in this study. The recurrence rate after the first conservative therapy has been reported in patients with PSP $(14,15)$; however, there have been no reports regarding the recurrence rate after the second conservative therapy, especially in young patients. For future studies, it would be beneficial to compare the recurrence rates after surgery and after second conservative therapy in young patients.

In conclusion, although young patients generally have a high rate of postoperative recurrence of PSP, the patients aged $<25$ years with $\mathrm{BMI} \geq 18$ and no contralateral bulla 
neogenesis have a significantly lower rate of postoperative recurrence rate.

\section{Acknowledgments}

None.

\section{Footnote}

Conflicts of Interest: The authors have no conflicts of interest to declare.

Ethical Statement: The authors are accountable for all aspects of the work in ensuring that questions related to the accuracy or integrity of any part of the work are appropriately investigated and resolved. The Institutional Review Board of Takasago Municipal Hospital approved this study (No. 2018-11), and we obtained informed consent from each patient by optout.

\section{References}

1. Baumann MH, Strange C, Heffner JE, et al. Management of spontaneous pneumothorax: an American College of Chest Physicians Delphi consensus statement. Chest 2001;119:590-602.

2. MacDuff A, Arnold A, Harvey J. Management of spontaneous pneumothorax: British Thoracic Society Pleural Disease Guideline. Thorax 2010;65 Suppl 2:ii18-31.

3. Tschopp JM, Bintcliffe O, Astoul P, et al. ERS task force statement: diagnosis and treatment of primary spontaneous pneumothorax. Eur Respir J 2015;46:321-35

4. Nakayama T, Takahashi Y, Uehara H, et al. Outcome and risk factors of recurrence after thoracoscopic bullectomy in young adults with primary spontaneous pneumothorax. Surg Today 2017;47:859-64.

5. Huang $\mathrm{H}$, Ji H, Tian H. Risk factors for recurrence of primary spontaneous pneumothorax after thoracoscopic surgery. Biosci Trends 2015;9:193-7.

6. Noh D, Lee S, Haam SJ, et al. Recurrence of primary spontaneous pneumothorax in young adults and children. Interact Cardiovasc Thorac Surg 2015;21:195-9.

7. Tsuboshima K, Wakahara T, Matoba Y, et al. Pleural coating by $50 \%$ glucose solution reduces postoperative recurrence for spontaneous pneumothorax. Ann Thorac Surg 2018;106:184-91.

8. Muramatsu T, Shimamura M, Furuichi M, et al. Cause and management of recurrent primary spontaneous pneumothorax after thoracoscopic stapler blebectomy. Asian J Surg 2011;34:69-73.

9. Cho S, Jheon S, Kim DK, et al. Results of repeated videoassisted thoracic surgery for recurrent pneumothorax after primary spontaneous pneumothorax. Eur J Cardiothorac Surg 2018;53:857-61.

10. Tsuboshima K, Nagata M, Wakahara T, et al. Association between postoperative bulla neogenesis at the staple line and resected lung weight for primary spontaneous pneumothorax: a retrospective study using the inverseprobability of treatment weighted method in patients grouped according to age. J Thorac Dis 2016;8:3676-81.

11. Tsuboshima K, Matoba Y, Wakahara T, et al. Natural history of bulla neogenesis for primary spontaneous pneumothorax: a propensity score analysis. Gen Thorac Cardiovasc Surg 2019;67:464-9.

12. Tsuboshima K, Nagata M, Wakahara T, et al. Feasibility of single-incision thoracoscopic surgery using a modified chest wall pulley for primary spontaneous pneumothorax: a propensity score matching analysis. Surg Today 2017;47:1129-34.

13. Katayama H, Kurokawa Y, Nakamura K, et al. Extended Clavien-Dindo classification of surgical complications: Japan Clinical Oncology Group postoperative complications criteria. Surg Today 2016;46:668-85.

14. Chen JS, Chan WK, Tsai KT, et al. Simple aspiration and drainage and intrapleural minocycline pleurodesis versus simple aspiration and drainage for the initial treatment of primary spontaneous pneumothorax: an open-label, parallel-group, prospective, randomised, controlled trial. Lancet 2013;381:1277-82.

15. Sadikot RT, Greene T, Meadows K, et al. Recurrence of primary spontaneous pneumothorax. Thorax 1997;52:805-9.

16. Tan J, Yang Y, Zhong J, et al. Association Between BMI and Recurrence of Primary Spontaneous Pneumothorax. World J Surg 2017;41:1274-80.

17. Onuki T, Kawamura T, Kawabata S, et al. Neo-generation of neogenetic bullae after surgery for spontaneous pneumothorax in young adults: a prospective study. J Cardiothorac Surg 2019;14:20.

Cite this article as: Tsuboshima $\mathrm{K}$, Matoba $\mathrm{Y}$, Wakahara T. Contralateral bulla neogenesis associated with postoperative recurrences of primary spontaneous pneumothorax in young patients. J Thorac Dis 2019;11(12):5124-5129. doi: 10.21037/ jtd.2019.12.05 